\title{
Apropiación de Sistemas de Tecnologías de la Información para toma de Decisiones de Productores Agroindustriales Basada en Videojuegos Serios. Una Revisión
}

\author{
Urbano E. Gómez-Prada ${ }^{(1,3) *}$, Martha L. Orellana-Hernández ${ }^{(2)}$ y Jesús M. Salinas-Ibáñez ${ }^{(3)}$ \\ (1) Facultad de Ingeniería de Sistemas e Informática, Universidad Pontificia Bolivariana (UPB), Autopista a Piedecuesta \\ Km 7, Edif. L Oficina 301, Bucaramanga, Colombia (e-mail: urbano.gomez@upb.edu.co) \\ (2) UNAB Creative, Universidad Autónoma de Bucaramanga (UNAB), Avenida 42 No. 48-11, Edificio L Piso 7 , \\ Bucaramanga, Colombia (e-mail: morellana@unab.edu.co) \\ (3) Instituto de Investigación e Innovación Educativa (IRIE), Universidad de las Islas Baleares (UIB), Calle de Miguel de \\ los Santos Oliver 2, Edif. Sa Riera, Palma, España (e-mail: jesus.salinas@uib.es) \\ ${ }^{*}$ Autor a quien debe ser dirigida la correspondencia
}

Recibido May. 30, 2019; Aceptado Jul. 2, 2019; Versión final Ago. 23, 2019, Publicado Oct. 2019

\section{Resumen}

Se presenta una revisión sistemática de estudios relacionados con temas de apropiación de tecnologías de la información (TI), dinámica de sistemas (DS), videojuegos serios (VS) y sistemas de información para la toma de decisiones (DSS), asociados a la agroindustria, realizada con base a la información entregada por la Web of Science y Scopus. Entre los resultados de esta revisión se destaca la baja adopción de TI en el sector, especialmente por parte de los pequeños productores. Se observa también interés especial por mitigar esa brecha, específicamente la del uso de los sistemas de información y de desarrollos para la comprensión y aprendizaje de modelos con DS y VS. Esta revisión sirve de base para futuros trabajos relacionados con la apropiación de TI en sistemas productivos rurales.

Palabras clave: Apropiación; tecnologías de la información; agroindustria; videojuego serio; sistemas de Información; toma de decisiones

\section{Appropriation of Information Technology Systems for Decision Making Support Systems by Agroindustry Producers Based on Simulation Models or Serious Games: A Review}

\begin{abstract}
A systematic review of studies related to topics of appropriation of IT, systems dynamics (DS), serious videogames (SG) and decision support systems (DSS) associated with agroindustry done by using the information provided by the Web of Science and Scopus. Among the results of this review is the low adoption of IT in the sector, especially by small producers. It was also observed special interest in mitigating that gap, specifically, on the use of information systems and developments for understanding and learning models with system dynamics and serious video games. This review serves as a basis for future work related to the appropriation of IT in rural productive systems.
\end{abstract}

Keywords: Appropriation, information technologies; agroindustry; serious video game; information systems; decision making 


\section{INTRODUCCIÓN}

La Apropiación de DSS en la Agroindustria es lenta y baja, debido a, la complejidad que perciben sus usuarios (Walmsley y Oddy, 2018) (En este documento, es asumida como la cantidad de interacciones o dependencias entre los elementos que componen un sistema (Andrade et al, 2001)), las pocas estrategias de adopción (Whittenbury y Davidson, 2009) y a la necesidad de modificar patrones de conducta (Molano, 2016). En Colombia, según Sierra (2017) se debe procurar apoyar el desarrollo rural, entregando mejor información al agricultor formándolo en prácticas agrícolas y según la Universidad Nacional de Colombia (2014) generando aprendizaje en los administradores sobre sus sistemas que les permitan participar, crecer en la economía y adoptar tecnología, la cual no se ha logrado en la medida esperada debido a barreras como el arraigo cultural, el desconocimiento de la tecnología, la falta de mejores prácticas, la ausencia de capacitación y la creencia de que como se opera, es suficiente para tomar buenas decisiones. En Argentina, Otta et al (2016) manifiestan la necesidad de evaluar la sustentabilidad acorde a condiciones sociales, económicas y productivas analizadas con indicadores ecológicos y económicos con el cual dejaron en evidencia limitaciones en capacitación, asociación, vías de comercialización, eficiencia productiva y dependencia a insumos externos.

Con estas afirmaciones coinciden Arévalo et al (2015) quienes agregan que, como consecuencia de no usar Tecnologías de la Información ( $\mathrm{TI}$ ) en los sistemas se generan errores o retrasos en las decisiones, desconocimiento de las pérdidas o ausencia de utilidades, desestimación o abandono y por ende migración a las ciudades. Aunque la presencia de las TI facilita controlar procesos organizacionales al otorgar información para mejorar la eficiencia del proceso, en el sector agropecuario, además de ello, agrega valor al brindar la posibilidad de trazabilidad desde la gestación del animal hasta la venta o consumo de los productos.

El desarrollo industrial en la agroindustria debe hacer uso de TI que permitan su fortalecimiento debido a la necesidad de aprovechar flujos de datos para transformarlos en información y pronóstico para tomar así mejores decisiones aumentando la producción y la comercialización (Fritz, et al, 2019). Los sistemas de monitoreo agrícola, a escala global y regional, tienen como objetivo proporcionar información actualizada a diferentes actores y tomadores de decisiones sobre la producción de alimentos para tener seguridad alimentaria. Según Hendrickson et al (2008), hay una gran heterogeneidad por parte de los productores en la forma de llevar los procesos y es importante hacer adopción de tecnologías informáticas para la trazabilidad; por ende, es necesaria la presencia de entidades que muestren las bondades de contar con información entre los productores. En función de ello, los autores desarrollaron un esquema jerárquico, generado en talleres, en donde organizaron principios, criterios e indicadores, para el desarrollo de un DSS agrícola que promueva la sostenibilidad, en sistemas sin control de recursos hasta los integrados dinámicamente.

En Estados Unidos se propone que las entidades investigativas participen más en proyectos que mejore los resultados del mercado agrícola a pequeña escala y que ofrezcan niveles de servicio adecuados en las áreas rurales (Salemik y Strijker, 2018). Algunos de los servicios que se requieren, son el acceso a TI, cuya ausencia disminuye la probabilidad de atraer industrias innovadoras o creativas (Mahasuweerachai et al, 2010). En el Reino Unido, Erdiaw y Khorshed (2016) expresan que, la vinculación de las asociaciones en el desarrollo rural atrae el interés por disminuir, con información que se obtiene a partir de TI, los problemas sociales y ambientales, pero estos no pueden ser abordados únicamente por empresas, gobierno o sociedad en forma aislada ya que se deben generar alianzas rurales para participar en la economía y la sociedad. Además, Koutsouris (2006) expresa como desafío, la ayuda que se debe brindar a las comunidades rurales con el desarrollo de nuevas tecnologías, habilidades, actitudes, prácticas y formas de colaboración. En ese sentido, Morris et al (2017) manifiestan que la agroindustria enfrenta desafíos debido a reformas políticas para las economías rurales orientadas a mitigar problemas adicionales a los de producción de alimento generando incertidumbres y ambigüedades por la necesidad de adopción de TI. De manera similar, Bosworth afirma que las aplicaciones son cada vez más diversas y por ello la complejidad de la inclusión digital no radica en si se tienen herramientas sino en si se usan (Bosworth, 2010). La alta brecha digital en el sector requiere prácticas que muestren beneficios derivados de la adopción y del apoyo político para mitigarla (Warren, 2002)

En Brasil y Australia Vinholis et al y Park manifiestan la importancia de permitir herramientas de acceso a la información especializada para hacer trazabilidad de los sistemas productivos (y recomiendan, migrar a sistemas intensivos) y la capacitación del capital humano, como elementos importantes en la decisión de adopción (Vinholis et al, 2017). A pesar de una creciente implementación de las TIC, las comunidades rurales presentan problemas de exclusión y por ende hay una brecha arraigada debido a la lejanía de los sistemas productivos y los factores sociodemográficos como los niveles educativos y la situación laboral, para mitigarlos son necesarios factores de la oferta de infraestructura y de oportunidades de empleo (Park, 2017).

Para mayor claridad de la presentación que sigue es necesario presentar las definiciones de los conceptos base de la revisión: apropiación de TI, agroindustria y ganadería, SI, DSS, MS, DS y VS. 


\section{Apropiación de Tecnologías de la Información}

La apropiación de TI es la capacidad o competencia para usar las TI en pro de generar cambios en la forma de llevar a cabo procesos para mejorar la calidad y por ende los resultados productivos (Daza et al, 2017), al respecto, Álvarez y Bernal (2017) manifiestan la necesidad de trabajar en estrategias para la apropiación de TI que generen confianza y conocimiento en estas tecnologías, ya que en países desarrollados, tecnologías como la simulación o los sistemas expertos son usadas frecuentemente en la innovación y evaluación de procesos y en el desarrollo de cultura organizacional.

Somers y Stapleton (2014) expresan la apropiación como un reto para el diseño de la tecnología a partir de la respuesta a cambios en el trabajo y en las condiciones de vida y que deben tener equilibrio entre el manejo de personal, los beneficios a la comunidad y los factores técnicos, es decir tener un enfoque sistémico con interacción de aspectos físicos, cognitivos y emocionales del usuario.

\section{Agroindustria y Ganadería}

La agroindustria es una actividad económica enfocada en la producción, industrialización y comercialización de productos agropecuarios o naturales (Liu et al, 2013). La ganadería es una rama de la agroindustria dedicada a la producción de carne y/o leche, la cual depende fundamentalmente de la calidad y disponibilidad de los pastos (Poder Agropecuario, 2017).

\section{Sistemas de Información (SI) y Sistemas de Información para la toma de decisiones (DSS)}

Los SI son un conjunto de varios componentes orientados a la gestión de datos e información, organizados para su uso y que fueron generados para un objetivo (Pressman, 2014). Una especialización de los SI, son los DSS, los cuales están enfocados en la toma de decisiones por parte de la alta dirección y ofrecen alternativas de solución a variados problemas (Budiyanto, 2017)

\section{Modelos de Simulación (MS) y Dinámica de Sistemas (DS)}

Los MS son representaciones de fenómenos o situaciones para simular eventos que pueden presentarse y tener herramientas de apoyo para tomar decisiones a partir de la credibilidad que tienen según su cercanía matemática con la realidad (Tarifa, 2001). Una metodología para hacer MS es la DS, la cual está compuesta por lenguajes que representan la estructura, generan ecuaciones en diferencias y grafican comportamientos de variables seleccionadas para analizar resultados en diferentes escenarios (Andrade et al, 2001). Andrade et al (2012) presentan Pesco, un MS en DS para el aprendizaje sobre producción piscícola que permite desarrollar competencias para la toma de decisiones y que busca identificar el conocimiento que se genera con él en cuanto a la crianza de peces en los niños de escuelas rurales.

\section{Videojuegos Serios (VS)}

Los VS son instrumentos interactivos digitales orientados al entretenimiento y el aprendizaje (Schell, 2014) que generan experiencias para la educación, la competitividad y la colaboración buscando que haya transferencia de conocimiento y apropiación de la sustentabilidad y responsabilidad social (Victoria et al, 2017) y que tienen características como la opción de controlar diferentes variables, tomar decisiones, establecer estrategias y comparar efectos de sus acciones con niveles de aprendizaje en un entorno dado (Gros, 2009). Deben ofrecer reglas acordes al sistema que representan para obligar a entenderlo y avanzar al siguiente nivel (López, 2016). Para optimizarlos y mitigar limitaciones o problemas de su aplicación, según González (2014) deben buscar transformaciones sociales, mejorar el rendimiento laboral y disminuir la brecha digital, entre otros. Un ejemplo, es SAMI, un VS para el aprendizaje de sistemas de producción bovina soportado en un MS en DS en donde se simulan decisiones a tomar en una finca ganadera (Gómez y Gómez, 2017).

\section{METODOLOGIA}

La revisión sistemática es una forma de investigación para recolectar, organizar, evaluar y sintetizar evidencias sobre temas específicos, realizada de acuerdo a un diseño preestablecido con la intención de responder a una pregunta de investigación (Velázquez, 2014). En este trabajo se hizo una revisión en Scopus (SC) y Web of Science (WoS), calificados por Velázquez como fuentes académicas internacionales de gran reconocimiento. La pregunta que responde el trabajo, gira en torno a la utilización, en la agroindustria, del modelado con DS y los VS, como elementos usados en estrategias de formalización de conocimiento, o generadores de apropiación de SI o DSS en los productores, o como apoyo para desarrollar DSS. 
Se buscaron fuentes de los últimos cinco años, cuya ecuación de búsqueda involucrara con el operador AND, cuatro términos usando Agricultura o Ganadería, Sistemas de información, Simulación y Videojuegos serios, en el título, abstract y palabras clave, en español e inglés, pero no hubo resultados. Entonces se extendió a los últimos doce años combinando términos afines a los mencionados obteniendo más de diez mil, por ende, se afinó la búsqueda con los términos: toma de decisiones y apropiación o adopción. Las expresiones de búsqueda y la cantidad de fuentes obtenidas en cada base de datos son presentadas en la Tabla 1 (Las mismas búsquedas se realizaron con las expresiones en español y allí son presentadas).

Tabla 1. Expresiones de búsqueda y resultados obtenidos

\begin{tabular}{|c|l|c|c|c|c|}
\hline \multirow{2}{*}{ ID } & Expresión & \multicolumn{2}{|c|}{ Inglés } & \multicolumn{2}{c|}{ Español } \\
\cline { 3 - 6 } E1 & $\begin{array}{l}\text { (agriculture OR cattle) AND ("system dynamics" OR simulation OR } \\
\text { "serious game*” OR “video game*” OR “computer game*” OR } \\
\text { "information system*” OR “decision support system*”) }\end{array}$ & 17526 & 11562 & 1245 & 4590 \\
\hline E2 & E1 AND ("decision making") & 1285 & 553 & 12 & 172 \\
\hline E3 & E1 AND (adoption OR appropriation) & 357 & 251 & 5 & 21 \\
\hline
\end{tabular}

De los resultados obtenidos fueron seleccionados, en un primer filtro, 314 trabajos usando como criterios de inclusión que describieran aspectos metodológicos y que tuviesen al menos una citación (este último, para los anteriores a 2019). En un segundo filtro, se seleccionaron los trabajos que además mostraran el desarrollo del componente o los resultados de adopción, seleccionando 76 trabajos. En un tercer filtro se seleccionaron 28 trabajos incluyendo aquellos que presentan la sistematización de la experiencia y excluyendo los que no tienen la intención de propender por la Adopción o Apropiación de la tecnología.

\section{RESULTADOS}

Se presenta un resumen de las fuentes seleccionadas según la metodología mencionada y que por tanto tienen relación con Apropiación de Tl y Agroindustria.

\section{Sistemas de Información (SI) y Sistemas de Información para la toma de decisiones (DSS)}

Aunque existen herramientas que mejoran los procesos agroindustriales y fortalecen el conocimiento en los agricultores, hay ausencia de interés en su adopción; por ende existe una necesidad de investigaciones que amplíen el enfoque hacia la apropiación, como por ejemplo, el DSS presentado por Whittenbury y Davidson (2009), para el manejo eficiente del agua y el apoyo de decisiones en cuanto a la optimización del riego según el crecimiento de las plantas, el cual contempla los factores socioculturales y estructurales.

Al desarrollar un DSS se deben tener en cuenta los contextos sociales para identificar las estrategias cognitivas con las que el sistema debe contar. Un DSS que se ajusta a esta característica es CropSAT, el cual fue desarrollado para revisar las tasas de fertilización del suelo, apoyar procesos de aprendizaje de los agricultores y generar estrategias que amplíen su cobertura (Lundström y Lindblomb, 2018). Acorde a ello es presentado por Mir y Padma (2018), DSSAT, el cual apoya las decisiones en cuanto al uso del suelo y comprende MS para más de cuarenta cultivos, viene siendo desarrollado desde 1989 y permite el análisis económico y la evaluación de riesgos en función del crecimiento de cultivos según entradas sobre características de variedad, condición del suelo, clima y fertilizantes. Según los autores, el DSS cuenta con criterios de adaptación al entorno e involucró a los usuarios en el proceso de desarrollo (característica que aumenta la adopción pero que se da muy pocas veces en el ámbito de SI orientados al agro).

Los DSS deben brindar alternativas sobre los modelos de conocimiento para apoyar en la identificación y resolución de problemas y más aún en el sector agroindustrial (Budiyanto, 2017); afín a ello, este autor presenta un DSS que busca aumentar la tasa de éxito de la inseminación artificial, sugiriendo la preparación adecuada según el ciclo estral del ganado (es decir, el crecimiento del folículo en el ovario); para el desarrollo hubo apoyo de los productores en la formalización de los indicadores del animal. La incorporación de modelado en los DSS brinda la oportunidad de integrar el conocimiento de varios productores en la toma de decisiones, cuando el conocimiento que se involucra en el DSS se hace manera fragmentada puede darse una baja adopción, pues genera entre los usuarios la percepción de gran complejidad con los requisitos de datos de entrada (Walmsley y Oddy, 2018). En particular, los autores presentan un DSS que apoya las dietas alimenticias para los animales según el crecimiento desde el destete en comparación con el peso de preñez.

Para apoyar el control y realizar trazabilidad en sistemas de producción lechera de bovinos y búfalos, Ponnusamy et al (2016) presentan un sistema experto, en donde probaron la ganancia de conocimiento y adquisición de habilidades de 140 agricultores seleccionados en dos pueblos de India. El estudio mostró que los agroindustriales obtuvieron aprendizajes más altos en comparación con los productores marginales debido 
a su inclinación por emprender la ganadería en líneas comerciales y a la generación de restricciones y sugerencias para mejorar su uso. Alawneh y Olchowy (2018) hacen alusión a un DSS para mejorar la producción lechera a partir de organizar los sistemas por la complejidad entre salud y productividad y evitar que las decisiones se basen solo en la experiencia. Los autores manifiestan la necesidad que hay, de pasar de la forma tradicional a la que sugiere el DSS, es decir, a la de comprender integralmente los problemas de salud y productividad mediante accesos oportunos a la información para mejorar el desempeño del hato.

También para producción lechera, Jago et al (2013) proponen un DSS de gestión de precisión que facilitan las decisiones y mejoran la eficiencia ya que los empleados se dedican a la operación y la recopilación de datos sobre animales y recursos, en las fases de producción, se da con sensores. Para hacer pronósticos en granjas lecheras, Vaillant y Baldinger (2016) desarrollaron SOLID-DSS, una librería de código abierto que cubre vacas lecheras y animales jóvenes y proporciona rutinas para simular el hato, la producción, los requisitos de energía y proteínas, la evaluación alimentaria y el crecimiento animal, la cual fue desarrollada para ser usada independientemente por cualquier DSS en la web. Por otra parte, en colaboración con productores de seis granjas lecheras para los desafíos de aprendizaje y adaptación, Eastwood et al (2012) presentan un DSS de precisión en donde se buscó la externalización del conocimiento tácito, el apoyo para revisar problemas de usabilidad y se determinó la importancia del apoyo de sensores para ingresar datos.

Los DSS deben presentar como mínimo, perspectivas financieras, procesos comerciales internos y externos, aprendizaje y crecimiento (Marimin y Kaneu, 2013), Marimin y Kaneu presentan un DSS desarrollado para aumentar la productividad sostenible en la gestión de la cadena piscícola que opera mediante alternativas de información del distrito de Southeast Maluku, Indonesia.

\section{Modelos de Simulación (MS) y Dinámica de Sistemas (DS)}

A pesar de que la agroindustria es considerada una actividad fundamental en el desarrollo de la economía de cualquier país (Basu y Sushanta, 2007), se deben desarrollar procesos de modernización para el crecimiento productivo, lo cual debe ir acompañado de políticas que lo fortalezcan (Liu et al, 2013). Basu y Sushanta describen un MS para mostrar la forma en que el crecimiento de capital y el aumento del producto interno bruto agrícola de un país disminuyen la pobreza; el MS fue generado gracias a la integración de dispositivos para ahorrar mano de obra. Por otra parte, Adenle et al presentan un MS realizado para enfatizar la relación entre los factores de riesgo que impiden el desarrollo de negocios agroindustriales en Nigeria y Tailandia, mostrando la importancia de la competitividad en mercados nacionales e internacionales, el cual busca la generación estatal de políticas para el desarrollo rural y la inversión agrícola en los países en desarrollo.

Para analizar las características en la comercialización de los productos de las cooperativas de comunidades agrícolas, Protil y Barreiros (2012) describen un MS en DS que representa los procesos críticos e involucra cuatro perspectivas tradicionales (financiera; clientes; proceso interno; crecimiento y aprendizaje) y agrega dos: la social y la de relación con los clientes. En cuanto a la dinámica del uso de la tierra para la ganadería, Siregar et al (2018) describen un MS en DS que estima, a partir de ampliar el territorio dedicado a la producción agrícola y facilitar el acceso a las tecnologías, el crecimiento económico de Kalimantan Occidental y el aumento en la producción de alimento; el modelo promueve cambios en la política central del gobierno de Indonesia con tecnologías de apoyo a los productores. También en producción de alimento, Shikuku (2017) presenta un MS para priorizar estrategias productivas según escenarios generados por la variación del clima para apoyar decisiones sobre la dedicación de la tierra que mejoren el retorno de la inversión y evaluar impactos de las tecnologías agrícolas y la factibilidad de la adopción.

En modelos poblacionales de ganadería, se encuentra AgroDiSi, un proyecto construido con DS enfocado al aprendizaje, la comprensión, la explicación, la intervención y la experimentación, el cual brinda lineamientos para construir MS en DS que incluyen los subsistemas etarios, biofísicos, financieros y productivos (Gómez et al, 2015). AgroDiSi se basó en SIPROBI y SIPROB, modelos para ganadería intensiva y extensiva que muestran algunas diferencias productivas, como por ejemplo la tasa de natalidad, la producción de leche, el tamaño de potreros, el personal requerido para la administración, entre otros (Gómez y Andrade, 2010).

De manera similar, Toledo et al (2018) presentan un MS en DS para la comprensión también del sistema bovino y sus interacciones con el entorno, en el cual se observa el estímulo del ternero en las funciones maternas; además contemplan algunos factores ecológicos que afectan el rendimiento y suben los costos. En ese sentido, pero para producción porcina, Stygar (2018) describe un MS que simula el crecimiento y variaciones en el peso corporal de los cerdos y que brindará en una siguiente fase, la opción de integrarse con un DSS, una herramienta de monitoreo que operará con datos reales para la toma de decisiones.

CROPWAT es un MS para producción de alimento en escenarios secos y normales que involucra aspectos de riego en pro de mejorar el rendimiento de las gramíneas forrajeras (Ruiz, 2018). En esa misma área, 
Andrieu et al (2015) presentan un MS para analizar posibilidades de intervención en la preparación de alimento para el engorde de ganado a pequeña escala en función de reglas decisivas frente a cambios climáticos y económicos que mejoren el rendimiento.

Para simular las dinámicas de la producción lechera en pastoreo, es presentado MINDY, un MS, el cual, según el patrón de pastoreo diurno de una vaca lechera y basado en la digestión y el metabolismo, las fluctuaciones diurnas debidas a estímulos funcionales de alimentación y características del animal como altura, densidad aparente y del alimento, la materia seca, composición y calidad de forraje, permite generar escenarios según patrones de preferencia y selección de los animales para la gestación que se puedan utilizar para elegir la dieta de los animales (Gregorini et al, 2015).

En cuanto a producción bovina, Bewley et al (2009) presentan un MS del estado productivo del animal que parte de un puntaje según la condición corporal obtenida tras analizar los puntos anatómicos, el cual fue desarrollado como estrategia de apoyo en nutricional, genética y bienestar como soporte a la toma de decisiones administrativas. De manera similar, Dowson et al (2019) describen POWDer, un MS en tiempo discreto para la optimización en la producción de leche para experimentar escenarios económicos y climáticos según el precio previsto y la cobertura de alimento, y, por ejemplo, analizar el impacto que se tendría en caso de reducir el almacenamiento, entre otros. En el modelo se obtienen resultados cercanos a los promedios reales de casos específicos de Nueva Zelanda.

\section{Videojuegos Serios (VS)}

Para desarrollar prácticas, tecnologías y enfoques innovadores en los ámbitos de los pequeños productores agrícolas y pecuarios fue desarrollado Highland Farmer, un VS desarrollado en Tanzania que busca generar conciencia en legisladores sobre los volúmenes de información que se dan ante los variados problemas en el uso de tierra de África Oriental (Radwan et al, 2016).

En ese sentido, y para explorar decisiones de uso de la tierra, específicamente para maíz en contextos socio ecológicos de siete poblaciones de la República Democrática Popular Lao, se desarrollaron juegos serios con el apoyo de los productores aprovechando que los VS proporcionan un procedimiento sistemático para ampliar, generalizar y afianzar el conocimiento y la comprensión del cultivo con la posibilidad de generar alternativas para la adopción, expansión, diversificación y abandono (Ornetsmüller et al, 2018).

Además, en cuanto al uso de la tierra, pero agregando factores climáticos, Fisher et al (2012) presentan un VS de administración agrícola usado en talleres con agricultores, productores, asesores e investigadores para tomar decisiones similares a las de una granja real en cuanto a capital y logística; con ello los autores expresan que los VS pueden ser una solución a desafíos agrícolas tales como la transferencia de conocimiento y el aumento de la productividad. Integrando la parte agrícola y la pecuaria es presentado Dynamix, un VS en donde los jugadores hacen intercambios entre granjas y crean relaciones de organización colectiva, escenarios posibles en logística de compras y ventas (Ryschawy et al, 2018).

\section{DISCUSIÓN}

La tabla 2 presenta por orden de aparición, el autor, el año de publicación, el país, el ámbito de aplicación del trabajo, un indicador para cada herramienta utilizada (SI/DSS, MS, DS, VS, en la intersección de cada fila con la columna de cada término se presenta el número uno para identificar la pertenencia; en la columna DS están los MS en DS) y por último un contador de estas herramientas en un mismo trabajo.

La tabla 3 presenta el total de trabajos agrupados por país de publicación y la tabla 4 muestra la cantidad por año; se observa que Estados Unidos es el país donde hay más publicaciones y que 2018 ha sido hasta ahora, el año más relevante, aunque cabe mencionar que 2019 aún no ha terminado.

Se aprecia que el ámbito con más trabajos (12) es el de producción bovina seguido de uso de la tierra (7). (aunque de los primeros doce, tres se especializan en temas de inseminación y dietas o se mezclan con búfalos, además ocho son específicos en producción de leche). Hay pocos trabajos (4) sobre producción agrícola (aunque uno la combina con producción pecuaria), producción de alimento para animales (2), sistemas productivos porcinos (1), piscícolas (1) y uso eficiente del agua (1).

En resumen, fueron encontrados:

1.- Once DSS de los cuales, solo cuatro se apoyan en MS y solo dos son para producción bovina.

2.- Dieciocho MS de los cuales solo cuatro son en DS y solo uno sirvió de apoyo para construir un VS.

3.- Cuatro VS, en donde tres estaban orientados a uso de la tierra y uno a producción agrícola y pecuaria. 
Tabla 2. Ámbito del sistema de producción agrícola y tecnología utilizada por autor

\begin{tabular}{|c|c|c|c|c|c|c|c|c|c|}
\hline$\#$ & Autor & Año & País & Ámbito de Aplicación & SI/DSS & MS & DS & VS & Contador \\
\hline 1 & Whittenbur y Davidson & 2009 & Reino Unido & Uso eficiente del agua & 1 & & & & 1 \\
\hline 2 & Lundström y Lindblomb & 2018 & Estados Unidos & Uso de la tierra & 1 & 1 & & & 2 \\
\hline 3 & Mir y Padma & 2018 & Reino Unido & Uso de la tierra & 1 & 1 & & & 2 \\
\hline 4 & Budiyanto & 2017 & Reino Unido & Prod. bovina - Inseminación & 1 & & & & 1 \\
\hline 5 & Walmsley y Oddy & 2018 & Australia & Prod. bovina - dietas & 1 & 1 & & & 2 \\
\hline 6 & Ponnusamy & 2016 & India & Prod. bovina y bufalina - leche & 1 & & & & 1 \\
\hline 7 & Alawneh y Olchowy & 2018 & Suiza & Prod. bovina - leche & 1 & & & & 1 \\
\hline 8 & Jago et al & 2013 & Australia & Prod. bovina - leche & 1 & & & & 1 \\
\hline 9 & Vaillant y Baldinger & 2016 & Holanda & Prod. bovina - leche & 1 & 1 & & & 2 \\
\hline 10 & Eastwood et al & 2012 & Estados Unidos & Prod. bovina - leche & 1 & & & & 1 \\
\hline 11 & Marimin y Kaneu & 2013 & Indonesia & Prod. Piscícola & 1 & & & & 1 \\
\hline 12 & Basu y Sushanta & 2008 & Estados Unidos & Prod. agrícola & & 1 & & & 1 \\
\hline 13 & Liu et al & 2013 & Italia & Prod. Agrícola & & 1 & & & 1 \\
\hline 14 & Protil y Barreiros & 2012 & Suiza & Prod. Agrícola & & & 1 & & 1 \\
\hline 15 & Siregar et al & 2018 & Indonesia & Uso de la tierra & & & 1 & & 1 \\
\hline 16 & Shikuku & 2017 & Estados Unidos & Uso de la tierra & & 1 & & & 1 \\
\hline 17 & Gómez et al & 2015 & Chile & Prod. Bovina & & & 1 & & 1 \\
\hline 18 & Toledo et al & 2018 & Estados Unidos & Prod. Bovina & & & 1 & & 1 \\
\hline 19 & Stygar & 2018 & Reino Unido & Prod. Porcina & & 1 & & & 1 \\
\hline 20 & Ruiz & 2011 & México & Prod. de alimento para animales & & 1 & & & 1 \\
\hline 21 & Andrieu et al & 2015 & Estados Unidos & Prod. de alimento para animales & & 1 & & & 1 \\
\hline 22 & Gregorini et al & 2015 & Australia & Prod. bovina - leche & & 1 & & & 1 \\
\hline 23 & Bewley et al & 2015 & Estados Unidos & Prod. bovina - leche & & 1 & & & 1 \\
\hline 24 & Dowson et al & 2019 & Nueva Zelanda & Prod. bovina - leche & & 1 & & & 1 \\
\hline 25 & Radwan et al & 2016 & Canadá & Uso de la tierra & & & & 1 & 1 \\
\hline 26 & Ornetsmüller et al & 2018 & Canadá & Uso de la tierra (maíz) & & & & 1 & 1 \\
\hline 27 & Fisher et al & 2012 & Australia & Uso de la tierra (clima) & & & & 1 & 1 \\
\hline 28 & Ryschawy et al & 2018 & Francia & Prod. agrícola y pecuaria & & 1 & & 1 & 2 \\
\hline \multicolumn{5}{|r|}{ Total } & 11 & 14 & 4 & 4 & 33 \\
\hline
\end{tabular}

Tabla 3. Cantidad de trabajos por país

\begin{tabular}{|c|c|c|}
\hline \multicolumn{3}{|c|}{ Resumen por País } \\
\hline País & Cantidad & \% de Participación \\
\hline Estados Unidos & 7 & 0,25 \\
\hline Australia & 4 & 0,14 \\
\hline Reino Unido & 4 & 0,14 \\
\hline Canadá & 2 & 0,07 \\
\hline Indonesia & 2 & 0,07 \\
\hline Suiza & 2 & 0,07 \\
\hline Chile & 1 & 0,04 \\
\hline Francia & 1 & 0,04 \\
\hline Holanda & 1 & 0,07 \\
\hline India & 1 & 0,04 \\
\hline Italia & 1 & 0.04 \\
\hline México & 1 & 0,04 \\
\hline Nueva Zelanda & 1 & 0,04 \\
\cline { 2 - 3 } & 28 &
\end{tabular}

Tabla 4. Cantidad de trabajos por año

\begin{tabular}{|c|c|c|}
\hline \multicolumn{3}{|c|}{ Resumen por año } \\
\hline Año & Cantidad & \% de Participación \\
\hline 2019 & 1 & 0,04 \\
\hline 2018 & 9 & 0,32 \\
\hline 2017 & 2 & 0,07 \\
\hline 2016 & 3 & 0,11 \\
\hline 2015 & 4 & 0,14 \\
\hline 2013 & 3 & 0,11 \\
\hline 2012 & 3 & 0,11 \\
\hline 2011 & 1 & 0,04 \\
\hline 2009 & 1 & 0,04 \\
\hline 2008 & 1 & 0,04 \\
\hline \multicolumn{2}{|c|}{} \\
\cline { 2 - 3 } & 28 & \\
\cline { 2 - 3 }
\end{tabular}




\section{CONCLUSIONES}

De acuerdo con el trabajo presentado y los resultados obtenidos, se pueden plantear las siguientes conclusiones principales:

1.- Se encontraron coincidencias sobre el beneficio del uso de las herramientas de TI para favorecer la apropiación y fortalecer la trazabilidad de los procesos productivos. Se destaca la importancia de incluir técnicas de pronóstico en los subsistemas administrativos, demográficos, productivos, ambientales, sociales, de uso de tierra y de crecimiento económico, para obtener mejores resultados al permitir una toma de decisiones basada en el análisis de la productividad y no solo en la experiencia.

2.- Se encontraron experiencias de implementación de sistemas de TI para representar los sistemas, formalizar el conocimiento y gestionar los procesos productivos, aunque, para fortalecer la apropiación de los DSS, no se usaron VS, a pesar de que brindan experiencias de transferencia de conocimiento y apropiación a los usuarios de los sistemas productivos,

3.- Se presenta una oportunidad de utilizar los VS para apoyar estrategias de apropiación de DSS en producción bovina aprovechando beneficios que ofrecen tales como apoyar la comprensión, la generación de conciencia sobre la cantidad de datos que surgen en la operación y la realización de experimentos de análisis en escenarios. Además, sería pertinente soportar el desarrollo tanto del DSS como del VS, en MS para unificar el conocimiento favoreciendo la representación de los procesos y la identificación y análisis de estrategias productivas.

\section{REFERENCIAS}

Alawneh, J., y W. Olchowy, Functionality and Interfaces of a Herd Health Decision Support System for Practicing Dairy Cattle Veterinarians in New Zealand, doi: 10.3389/fvets.2018.00021, Frontiers in Veterinary Science, 5 (21) (2018)

Álvarez, E., y C. Bernal, Modelo de innovación abierta: Énfasis en el potencial humano, doi: 10.4067/S071807642017000100007, Información tecnológica, 28(1), 65-76, Chile (2017).

Andrade, H., G. Maestre, J. Castro J y M. Zambrano, Aprendiendo con Dinámica de Sistemas y desarrollando competencias para la toma de decisiones: Una experiencia en la educación: Ambiente Virtual de Aprendizaje Pesco 2.0. Décimo Congreso Latinoamericano de Dinámica de Sistemas. Buenos Aires, Argentina (2012).

Andrade, H., I. Dyner y otros tres autores. Pensamiento Sistémico, Diversidad en búsqueda de unidad. UIS. Bucaramanga, Colombia (2001).

Andrieu, N., K. Descheemaeker y T. Sanou, Chia, E. Effects of technical interventions on flexibility of farming systems in Burkina Faso: lessons for the design of innovations in West Africa, doi: 10.1016/j.agsy.2015.02.010, Agricultural Systems, 136, 125-137 (2015).

Arévalo, J., R. Bayona R y D. Rico, El problema de la brecha tecnológica: un asunto de cultura. Revista Sinapsis. 7 (7), 35- 43 (2015).

Basu, S., y S. Mallick, When does growth trickle down to the poor? The Indian case. doi: 10.1093/cje/bem053, Cambridge Journal of Economics, 32 (3), 461-477 (2008)

Bewley, J., y M. Schutz, Potential of using new technology for estimating body condition scores. 18th annual tri-state dairy nutrition conference, S823.91-532, Fort Wayne, Indiana, Estados Unidos. (2009).

Bosworth, G. Commercial counterurbanisation: an emerging force in rural economic development, doi: 10.1068/a42206, Environ, 42 (4), pp. 966-981 (2010).

Budiyanto, C., Decision Support Systems Development for an Artificial Insemination Project in Community Based Precision Livestock Farming. doi:10.1088/1757-899X/180/1/012242, IOP Conference Series: Materials Science and Engineering. IOP Publishing, Reino Unido (2017).

Daza, L., C. Hernández, A. Quijano y J. Serna, Plan estratégico del Departamento de Santander. Acceso 27 de noviembre (2018) goo.gl/4fSpDW. Colombia (2017).

Dowson, O., A. Philpott y A. Mason, Downward, A. A multi-stage stochastic optimization model of a pastoral dairy farm, doi: 10.1016/j.ejor.2018.10.033, European Journal of Operational Research, 274 (3), 1077-1089. (2019).

Eastwood, C., D. Chapman y M. Paine, Networks of practice for co-construction of agricultural decision support systems: case studies of precision dairy farms in Australia, doi: 10.1016/..agsy.2011.12.005, Agricultural Systems, 108, 10-18. (2012)

Erdiaw, M., y A. Khorshed, Towards understanding digital divide in rural partnerships and development: A framewor and evidence from rural Australia, doi: 10.1016/j.jurstud.2015.12.002, Rural Studies, 43, 214-224 (2016)

Fisher, J., I. Farré y otros tres autores. Serious games to explore uncertainty of future farms. Proceedings of 16 th Agronomy Conference 2012, Australia (2012). 
Fritz, S., L. See, y otros cinco autores. A comparison of global agricultural monitoring systems and current gaps, doi: 10.1016/j.agsy.2018.05.010, Agricultural Systems, 168, 258-272 (2019).

Gómez, U. y H. Andrade, Propuesta de un modelo de simulación de ganadería intensiva bovina (SIPROBI). Congreso Latinoamericano de Dinámica de Sistemas. Chile (2010).

Gómez, U., H. Andrade y C. Vásquez, Lineamientos metodológicos para construir ambientes de aprendizaje en sistemas productivos agropecuarios soportados en dinámica de sistemas, doi: 10.4067/S0718-07642015000400016, Información Tecnológica, 25(4), 125-136. Chile (2015).

Gómez, U. y O. Gómez, Simulation of cattle farms with System Dynamics in a serious videogame. Case: SAMI. Conf. Inter. en Agro BigData y Sistemas para la toma de decisiones. Uruguay (2017).

González C. Videojuegos para la transformación social. Aportaciones conceptuales y metodológicas. Bilbao: Universidad de Deusto, España. (2014).

Gregorini P., J. Villalba, F. Provenza, P. Beukes y otro autor. Modelling preference and diet selection patterns by grazing ruminants: a development in a mechanistic model of a grazing dairy cow, MINDY, doi: 10.1071/AN14472, Animal Production Science, 55(3), 360-375 (2015).

Gros B. Certezas e interrogantes acerca del uso de los videojuegos para el aprendizaje, Comunicación, ISSN 1989-600X, 1(7), 251-264 (2009).

Hendrickson J., J. Hanson, D. Tanaka y G. Sassenrath, Principles of integrated agricultural systems: Introduction to processes and definition, doi: 10.1017/S1742170507001718, Renewable Agriculture and Food Systems, 23, 265-271 (2018)

Jago, J., C. Eastwood, K. Kerrisk e I. Yule, Precision dairy farming in Australasia: adoption, risks and opportunities, doi: 10.1071/AN12330, Animal Production Science, 53(9), 907-916. (2013).

Tarifa, E. Teoría de modelos y simulación. Fac. de Ingeniería de la Universidad de Jujuy, Argentina (2001).

Koutsouris, A. ICTs and Rural Development beyond the Hype, Journal of Extension Systems, ISSN: 0970-2989, 22(1), 46 (2006)

Liu, P., S. Koroma, P. Arias y D. Hallam, Trends and impacts of foreign investment in developing country agriculture: evidence from case studies. Food and Agriculture Org. of the United Nations, Roma, Italia. (2013).

López, C. El videojuego como herramienta educativa. Posibilidades y problemáticas acerca de los serious games. Apertura, 1(8) (2016).

Lundström, C., y J. Lindblom, Considering farmers' situated knowledge of using agricultural decision support systems (AgriDSS) to Foster farming practices: The case of CropSAT, doi: 10.1016/j.agsy.2017.10.004, Agricultural Systems, vol. 159, p. 9-20. (2018)

Mahasuweerachai, P, B. Whitacre y D. Shideler, Does broadband access impact migration in America? Examining differences between rural and urban areas, Regional Studios, ISSN 1553-0892, 5-26; 40(1) (2010)

Marimin Y, Teniwut K. Decision Support System for Increasing Sustainable Productivity on Fishery Agroindustry supply chain. International Conference on Advanced Computer Science and Information System. Indonesia (2013).

Mir S., y T. Padma, Generic Multiple-Criteria Framework for the development of agricultural DSS, doi: 10.1080/12460125.2018.1437501, Journal of Decision Systems, 26, 341-367. Inglaterra (2018).

Molano A. Tecnología para la agroindustria y las ciudades inteligentes en Colombia. Acceso 2 de enero (2019) goo.gl/j81vbY (2016).

Morris W., A. Henley y D. Dowella. Farm diversification, entrepreneurship and technology adoption: Analysis of upland farmers in Wales, doi: 10.1016/j.jurstud.2017.05.014, Rural Studies, 53, 132-143 (2017)

Ornetsmüller C., J. Castella y P. Verburg, A multiscale gaming approach to understand farmer's decision making in the boom of maize cultivation in Laos, doi: 10.5751/ES-10104-230235, Ecology and Society, 23(2) (2018).

Otta, S., J. Quiroz, E. Juaneda y otros tres autores, Evaluación de sustentabilidad de un modelo extensivo de cría bovina en Mendoza, Argentina, FCA Uncuyo, ISSN: 1853-8665, 48(1), 179-195 (2016).

Park, S., Digital inequalities in rural Australia: A double jeopardy of remoteness and social exclusion, doi: 10.1016/j.jrurstud.2015.12.018, Rural Studies, 54, 399-407 (2017).

Poder Agropecuario. Alimentación del ganado y sistemas de pastoreo. Acceso 7 de diciembre (2018) goo.gl/wxtehk (2017).

Ponnusamy K., N. Sriram y otros cuatro autores, Effectiveness of cattle and buffalo expert system in knowledge management among the farmers, Indian Journal of Animal Sciences, ISSN: 0367-8318, 86(5), 604-608 (2016).

Pressman R. Ingeniería del Software. Mc Graw Hill, 7ª Edición, México (2014).

Protil R., y R. Barreiros, Strategic Control of Agro-Industrial Cooperatives: A Strategic Map Proposal. 30th International Conference of the System Dynamics Society. St. Gallen, Switzerland (2012). 
Radwan R, N. Jad y L. Odeth, Mansour A. A serious Game for Agricultural Knowledge Development. Acceso 1 de febrero (2019) goo.gl/hJdTcE. (2016).

Ryschawy, J., A. Charmeau y otros tres autores. Using the serious game Dynamix to design trade relationships among grain and livestock farmers in Ariege, Fourrages, ISSN: 0429-2766 (235), 207-212 (2018).

Ruiz, O., R. Arteaga y otros tres autores. Irrigation requirements and yield prediction in forage grasses using a simulation model in Tabasco, México, Agrociencia, ISSN: 2521-9766, 45(7), 745-760 (2011).

Salemink, K., y D. Strijker, The participation society and its inability to correct the failure of market players to deliver adequate service levels in rural areas. doi: 10.1016/j.telpol.2018.03.013, Telecommunications Policy (2018).

Schell, J. The Art of Game Design: A Book of Lenses. 2 ${ }^{\mathrm{a}}$ Edición. Florida: CRC Press. Estado Unidos, (2014).

Shikuku, K., R. Valdivia y otros cinco autores. Prioritizing climate-smart livestock technologies in rural Tanzania: A minimum data approach, doi: 10.1016/j.agsy.2016.06.004, Agricultural systems, 151, 204-216 (2017)

Sierra J., Uso TIC agrícola: Ajustes en la cosecha. Acceso 9 de enero (2017) goo.gl/BpwrrLn (2016)

Siregar P., J. Supriatna, R. Koestoer y D. Harmantyo, System Dynamics Modeling of Land Use Change in West Kalimantan, Indonesia, doi: 10.11598/btb.2018.25.2.792, Biotropia, 25(2), 103-111. (2018).

Somers S., y L. Stapleton, e-Agricultural innovation using a humancentred systems lens, proposed conceptual framework, doi: 10.1007/s00146-013-0475-x, Al \& Society, 29(2), 193-202. (2014).

Stygar, A., K. Dolecheck y A. Kristensen. Analyses of body weight patterns in growing pigs: a new view on body weight in pigs for frequent monitoring. doi: 10.1017/S1751731117001690, animal, 12(2), 295-302 (2018).

Toledo L., T. Barcelos, M. Rodrigues y L. Ambrósio. Modelling the Dynamics of Cow-Calf Dyadic Behavior, doi: 10.4018/ijsda.2018100101, International Journal of System Dynamics Applications. 7(4), 19. (2018).

Universidad Nacional de Colombia. Agroindustria y comercio desaprovechan TIC. Acceso 29 de octubre (2018), goo.gl/YwQGxg (2014)

Vaillant, J., y L. Baldinger, Application note: An open-source JavaScript to simulate dairy cows and young stock, their growth, requirements and diets, doi:10.1016/j.compag.2015.11.005, Comp. and Electronics in Agriculture, 120, 7-9 (2016)

Velásquez, J., Una Guía Corta para escribir Revisiones Sistemáticas de Literatura, DYNA, ISSN 0012-7353, 81(187), 9$10(2014)$

Victoria, R., S. Utrilla y A. Santamaría, Aprendizaje basado en juegos. Una alternativa viable para la enseñanza significativa de la sustentabilidad, Revista sobre educación media y superior, ISSN: 2488 - 6507, 4(7) (2017)

Vinholis, M., M. Carrer y H. Souza, Adoption of beef cattle traceability at farm level in São Paulo State, Brazil., doi: 10.1590/0103-8478cr20160759, Cienc. Rural, 47(9). (2017).

Warren, M. Adoption of ICT in agricultural management in the United Kingdom: the intra-rural digital divide. Agric. Econ., 48, 2002 (1): 1-8. (2002)

Walmsley, B., y V. Oddy, Modelling systems to describe maternal productivity, with the aim of improving beef production efficiency by eliciting practice change, doi: 10.1071/AN14874, Animal production science, 58(1), 193-205 (2018)

Whittenbury, K., y P. Davidson. Beyond adoption: The need for a broad understanding of factors that influence irrigators' decision-making, doi: 10.5172/rsj.351.19.1.4, Rural Society. 19(1), 4-16 (2009) 